\title{
Additional Features of Food Induced Immediate Reaction in the Esophagus (FIRE) in a Series of Adult Patients with Eosinophilic Esophagitis
}

\author{
Mark Holbreich ${ }^{1}$ and Alex Straumann ${ }^{2}$ \\ ${ }^{1}$ Allergy and Asthma Consultants \\ ${ }^{2}$ Gastroenterological Pracitce and EoE Clinic
}

January 5, 2021

Additional Features of Food Induced Immediate Reaction in the Esophagus (FIRE) in a Series of Adult Patients with Eosinophilic Esophagitis

To the Editor

Eosinophilic esophagitis (EoE) is a chronic disease characterized clinically by symptoms related to esophageal dysfunction and histologically by eosinophil predominant inflammation. The predominant symptom in adults with active EoE is solid food dysphagia due to the high incidence of esophageal stricture formation. A quantitative tool has been developed to characterize the nature of these symptoms (1). Most typically patients complain of food going down slowly, food getting stuck and pain. These symptoms are most likely to occur during the passage of dense solid foods such as meats, bread and rice.

Recently a phenomenon with a different symptom referred to as FIRE (food induced immediate reaction in the esophagus) has been described in adults with EoE. (2). Patients with eosinophilic esophagitis describe these esophageal symptoms as distinct from dysphagia. The symptoms typically occur after the food has been swallowed with rapid onset esophageal discomfort often described as tightening or pressure but without the sense of dysphagia. A large series of Swiss patients with eosinophilic esophagitis were queried via a form mailed to their homes or in clinics concerning symptoms related an immediate reaction in the esophagus distinct from dysphagia (2). A significant portion (49\%) reported some form of esophageal discomfort related to eating. Although certain subject characteristics in this study such as younger age of onset of $\mathrm{EoE}$ and higher prevalence of allergies was noted, detailed clinical information on each subject was not available for review. Here in we described a series of patients with biopsy proven eosinophilic esophagitis who describe characteristic symptoms of FIRE following a specific food ingestion. For each selected FIRE patient a detailed chart review was conducted characterizing their demographics and EoE history (Table 1) as well as characteristics of their FIRE profile (Table 2). A number of features were noted in this group of 8 EoE patients as a previously unrecognized symptom complex. The onset of symptoms most often occurred within 1-60 minutes (median 3.5 minutes) after ingestion of the food. The duration of symptoms lasted from $<10$ minutes to $>6$ hours (median 120 minutes). Among the patients the description of discomfort was often similar consisting of a tightening or pressure in the mid sternum. The most common foods inducing symptoms were beans, melon and beer. The symptoms were significant enough that patients attempted to subsequently avoid these foods. All patients were able to distinguish between symptoms related to their pollen food allergy syndrome and FIRE.

The exact mechanism of this esophageal discomfort has not been elucidated. It appears distinct from pollen food allergy syndrome. It also appears different from EoE as uncommon foods such as banana, melon and avocado were capable of invoking symptoms, notably foods more commonly associated with oral 
allergy syndrome. However the strong association with atopy, the rapid onset of symptoms and symptoms distinct from dysphagia suggest a local possibly immunologic factor causing an immediate esophageal mucosal response. A careful history of FIRE symptoms should be part of the routine evaluation of patients with eosinophilic esophagitis. Further investigations will be required to help better understand the frequency and mechanism of this newly described phenomenon.

Conflicts of Interest

MH no conflicts, AS no conflicts

Mark Holbreich M.D.

Alex Straumann M.D.

1. Schoepfer AM, Straumann A, Panczak R, et al. Development and validation of a symptom-based activity index for adults with eosinophilic esophagitis. Gastroenterology 2014;147:1255-1266.

2. Biedermann, L, Holbreich, M, Atkins, D, et al. Food-induced immediate response of the esophagus-A newly identified syndrome in patients with eosinophilic esophagitis. Allergy . 2020; 00: 1- 9.

Corresponding author

Mark Holbreich M.D.

Allergy and Asthma Consultants

Indianapolis IN USA

mholbreich@comcast.net

\section{Hosted file}

FIRE Table 1.pdf available at https://authorea.com/users/387371/articles/502493-additionalfeatures-of-food-induced-immediate-reaction-in-the-esophagus-fire-in-a-series-of-adultpatients-with-eosinophilic-esophagitis

\section{Hosted file}

Table 2 FIRE PROFILE.pdf available at https://authorea.com/users/387371/articles/502493additional-features-of-food-induced-immediate-reaction-in-the-esophagus-fire-in-aseries-of-adult-patients-with-eosinophilic-esophagitis 